\section{El voluntariado corporativo y la responsabilidad social empresarial: una oportunidad en la lucha contra el estigma y el impulso de la inserción laboral de las personas con trastorno mental grave}

\section{Corporate volunteerism and corporate social responsibility: a chance to counter the stigma of persons affected by a serious mental illness and to promote their labour integration}

\section{Palabras clave}

Voluntariado corporativo, trastorno mental grave, estigma, inserción laboral, responsabilidad social.

\section{Keywords}

Corporate volunteerism, serious mental ilness, stigma, labour integration, corporate social responsibility.

\section{Introducción}

El voluntariado corporativo (en adelante, VC) es una modalidad específica de voluntariado que se desarrolla en la empresa. Es el resultado de la combinación del voluntariado tradicional, que muestra el deseo individual de las personas de colaborar a favor de aspectos sociales o medioambientales, con la creciente demanda de una mayor responsabilidad por parte de las empresas en el contexto en el que lleven a cabo su actividad (VV. AA., 2006:37-38; García, 20I 2:295; Actualidad Económica, 20II). El VC es, pues, una faceta de la responsabilidad social en la empresa. El VC debe entenderse como un importante factor de creación de reputación externa positiva, ya que da una imagen de empresa responsable y, asimismo, mejora la percepción de la empresa a nivel interno al permitir mejorar el clima de trabajo, reforzar la cohesión de los empleados y el trabajo en equipo. De ello "se desprende que la mayoría de las empresas utiliza el VC como
Carlos de Fuentes

‘cdefuentes@manantialintegra.org»

Responsable Área Comercial

Manantial Integra Documenta

\section{Miguel Díaz}

〈mdiaz@fundacionmanantial.org〉

Director de Voluntariado y Ocio de la Fundación Manantial

\section{Marta Fernández de Avilés}

‘mfdeaviles@fundacionmanantial. org >

Educadora Social EASC* "Leganés"

\section{Saray Cobo}

〈scobo@fundacionmanantial.org〉

Técnico de Apoyo a la Inserción Laboral del CRL" "Torrejón de Ardoz"

\section{Susana Ruiz}

«sruiznovillo@fundacionmanantial. org>

Técnico de Apoyo a la Inserción Laboral del CRL" "San Blas"

\section{Raquel Herráiz}

«rherraiz@fundacionmanantial.org〉

Técnico de Apoyo a la Inserción Laboral del CRL" "San Blas"

\section{Juana Gómez}

‘jgomez@fundacionmanantial.org〉

Técnico de Apoyo a la Inserción Laboral CRL" "Fuenlabrada"

\footnotetext{
* Todos los CRL y el EASC pertenecen a la Red de Atención Social a Personas con Enfermedad Mental Grave de la Consejería de Asuntos Sociales de la Comunidad de Madrid.
}

\footnotetext{
Para citar:

De Fuentes, C. et al. (20I4): "El voluntariado corporativo y la responsabilidad social empresarial: una oportunidad en la lucha contra el estigma y el impulso de la inserción laboral de las personas con trastorno mental grave”, Revista Española de Discapacidad, 2 (2): 247-253.

$<$ http://dx.doi.org/IO.5569/234O5IO4.02.02.I4>
} 
una herramienta de recursos humanos" (Gómez et al., 20I 2:I I-I2), pudiéndose concluir que la acción social de las empresas ha pasado de la solidaridad a convertirse en una cuestión estratégica dentro de la gestión de personas de todas las empresas, sobre todo de las que tienen un tamaño medio o grande.

Como se sabe, en el VC existen tres actores principales: los empleados que realizan el voluntariado, la empresa que organiza tal actividad y una organización no lucrativa (ONL, que puede ser una ONG o una fundación). La esencia del VC es que sean prácticas en las que todas las partes implicadas recibirán beneficios por su realización. Dicho en la jerga típica del marketing, el VC es capaz de conseguir un winwin-win (Gallardo et al., 2010:58).

La contribución del presente trabajo radica en analizar el VC desde la perspectiva de la organización no lucrativa; en nuestro caso, exponiendo la experiencia de la Fundación Manantial, entidad creada en I995 dedicada a la atención integral de las personas con trastorno mental grave, que en la actualidad presta atención a cerca de 2.000 personas, con una plantilla de 285 trabajadores y habiendo constituido varios centros especiales de empleo en los que trabajan cerca de 200 trabajadores, más del $90 \%$ de ellos con trastorno mental grave.

Cuáles son los motivos por los que esta fundación comienza a implantar prácticas de VC con las empresas, la gestión de dicho proyecto y los resultados obtenidos tanto para la propia entidad como para el colectivo atendido, es el objeto de estas páginas. Existen numerosos trabajos que tratan la cuestión desde el ámbito de la empresa, pero son pocos los que lo analizan desde el prisma de la organización social (Comunidad de Madrid, 2008; FEVOCAM, 20I2), de ahí nuestro interés en aportar esta visión y experiencia.

\section{Voluntariado corporativo con personas con trastorno mental. Justificación del proyecto}

Tal como explica Alicia Molina, coordinadora de la Plataforma Antiestigma "UNO+" de la Fundación Manantial ${ }^{\mathrm{I}}$, los estereotipos más frecuentes expresados sobre las personas con enfermedades mentales son: peligrosidad, extrañeza, impredecibilidad, incapacidad para manejar su vida, incurabilidad, sentimiento de culpa, responsabilidad sobre lo que les ocurre, etc.

Las creencias anteriores pueden dar lugar a prejuicios o actitudes que reflejan la disposición de las personas para actuar de una forma negativa hacia el objeto del prejuicio y conllevan una conducta discriminatoria que tiene como consecuencia la privación de los derechos y la aparición de situaciones de especial dificultad para encontrar trabajo o vivienda, limitación en las relaciones personales, etc.

Desde Fundación Manantial compartimos la visión de Diego Lejarazu por la cual en el VC "los proyectos acometidos persigan cambios sociales, no sólo la realización de actividades puntuales" (Lejarazu, 20 2:27); en nuestro caso, en relación con las personas con trastorno mental grave, el verdadero y necesario cambio social es la lucha contra el estigma que sufre este colectivo.

Éste es, pues, el principal motivo para la puesta en marcha del proyecto de VC dentro de la Fundación Manantial. Se considera la responsabilidad social en la empresa en general y el VC en particular como medios idóneos para la lucha contra el estigma de las personas con trastorno mental grave y para la inserción laboral del colectivo, dado que las actividades se realizan con el apoyo del tejido empresarial. Si las empresas cambian su percepción en relación con los trabajadores que padecen un Trastorno Mental

I. Sobre esta plataforma, ver la información disponible en Internet en: ‘http://www.fundacionmanantial.org/uno_mas. php', acceso 25 de junio de 2014. 
y les conocen a través de actuaciones de VC, aumentan las posibilidades de que alguno de ellos pueda pasar a ser seleccionado en alguna empresa o que la empresa esté abierta a poder contratarles en un futuro. De hecho, eso ha ocurrido ya en la experiencia de nuestra fundación.

La causa principal del estigma hacia las personas con trastorno mental grave es la falta de información y la falta de experiencias personales que nos ayuden a tener otra imagen del colectivo. El VC es un vehículo idóneo para tener estas experiencias directas de contacto con personas con trastorno mental grave y cambiar la imagen negativa hacia el colectivo. Por este motivo colaboramos con las entidades con responsabilidad social en la empresa y los medios de comunicación, para que nos ayuden en nuestra misión y además difundir una imagen más objetiva y positiva de las personas con trastorno mental grave. Fundación Manantial facilita a entidades que lo soliciten los medios y las actividades necesarios para desarrollar prácticas de VC.

\section{La planificación del voluntariado} corporativo en Fundación Manantial

Siguiendo el trabajo de la Consejería de Familia y Asuntos Sociales de la Comunidad de Madrid (Comunidad de Madrid, 2008:I6) en Fundación Manantial comenzamos a planificar nuestro proyecto de VC analizando tres variables: por un lado, qué nos interesa hacer con la empresa; en segundo término, con qué empresas nos queremos relacionar y, en tercer lugar, con qué periodicidad podemos hacerlo y cuáles son las fórmulas de colaboración más idóneas. Veamos cada uno de estos aspectos con un poco más de detenimiento.

\subsection{Qué hacer con la empresa}

Éste es, sin duda, el aspecto clave del proyecto. Lo verdaderamente importante es que aquello que se haga tenga un valor añadido para las personas atendidas en Fundación Manantial.

Posibilidades de colaboración:

a. Actividades de un día de duración

- Una jornada laboral en un Centro de Rehabilitación Laboral (CRL). Un grupo de empleados voluntarios de empresa comparte un día trabajando codo con codo con personas con discapacidad por trastorno mental que se están preparando para acceder a un empleo. Es una experiencia que no deja indiferente y lleva a conocer al colectivo de una manera integral. Para las personas atendidas del CRL es una oportunidad para acercarse a una empresa importante y le ayudaría en su proceso de inserción laboral.

- Charlas vitales, donde empleados de la empresa puedan contar su experiencia personal y su relación con el empleo: búsqueda, formación, desarrollo profesional, etc. Sería especialmente interesante si las charlas las pudieran ofrecer personal de diferentes departamentos (recursos humanos, gestión, encargados, etc.).

- Visita de nuestros candidatos a la empresa.

- Visitas, excursiones o salidas culturales.

- Actividades deportivas conjuntas (partidos de fútbol, etc.).

- Actividades de educación ambiental: En colaboración con la empresa y con entidades de acción medioambiental se busca generar sinergias que favorezcan la sensibilización social, donde usuarios y voluntarios tienen un fin común, cuidar de la naturaleza.

b. Actividades de más de un día de duración

- Cursos específicos impartidos por empleados de la empresa para usuarios/as de los CRL. Por ejemplo, Excel, Access, Word, maquetación de 
documentos, jardinería, carpintería, limpieza u otras materias, a nivel grupal o individual.

- Quedamos: Proyecto de ocio y actividades culturales, enfocado a que las personas experimenten sentimientos de bienestar a la vez que innovan en su rutina habitual. Con carácter periódico se realizan salidas conjuntas. Los voluntarios pueden incorporarse a la/s salida/s que les resulten interesantes.

\subsection{Cuáles son las empresas potenciales para realizar el VC}

En un primer momento se apostó por aquellas que ya tenían experiencia en este ámbito y que contasen con personal específicamente dedicado a este cometido. De ahí que se seleccionaran las empresas, normalmente medianas o grandes, con un cierto bagaje y renombre en responsabilidad social en la empresa. Tal es el caso de la primera actuación de VC en Fundación Manantial, llevada a cabo junto con los voluntarios de "La Caixa" para realizar salidas de ocio con personas atendidas en un Equipo de Apoyo Social Comunitario de Leganés.

En un segundo momento, y tras tener algunas experiencias prácticas, se vio que cualquier empresa podía realizar actuaciones de responsabilidad social en la empresa provechosas para las personas atendidas en nuestra fundación. Es el caso, por ejemplo, de una pequeña productora de cine (La Pierna) con la que se llevaron a cabo videos realizados por las propias personas atendidas en un Centro de Rehabilitación Laboral del distrito madrileño de San Blas.

\subsection{Periodicidad y fórmulas de colaboración con empresas}

De las diferentes fórmulas para promover la participación de los empleados en las actividades de VC (Club de Excelencia en Sostenibilidad y Fundación Adecco, 2010:54-55; Sanz et al., 2OI2:99-IOI), Fundación Manantial opta por aquellas que están ligadas a la continuidad y al trabajo continuo entre empresa y organización no lucrativa. Aunque se trate de actividades puntuales, pensamos que es muy recomendable que se repitan en el tiempo. En las experiencias de VC de nuestra fundación, varias actividades son de un día de duración pero al repetirse todos los años se consigue una vinculación muy importante entre la empresa y nuestra entidad, repercutiendo de forma directa y muy beneficiosa en las personas con trastorno mental grave.

Tal como señala el estudio del Club de Excelencia en Sostenibilidad y Fundación Adecco (2010:26), uno de los aspectos relevantes que debe considerarse en los programas de VC "es que la identificación y selección de los campos de actuación y colectivos debe estar alineada con las actividades de relación con la sociedad que ya realice la empresa".

\section{Experiencias prácticas llevadas a cabo}

Contamos en nuestro haber con un cierto abanico de experiencias de voluntariado corporativo que ya se ha llevado y se llevan a la práctica de forma satisfactoria. Las resumimos en la Tabla I. 
Tabla 1. Experiencias prácticas

\begin{tabular}{|c|c|c|c|c|}
\hline Acciones & Formación & $\begin{array}{l}\text { Macroactividad: } \\
\text { carrera }\end{array}$ & Pro-Bono & Otros \\
\hline $\begin{array}{l}\text { - Taller de pintura. } \\
\text { Caixa. } \\
\text { - Actividades de } \\
\text { Ocio proyecto } \\
\text { "Quedamos". } \\
\text { Caixa. } \\
\text { - Actividades } \\
\text { culturales. BBVA. } \\
\text { - Jornadas de } \\
\text { trabajo. AXA y } \\
\text { Telefónica. }\end{array}$ & $\begin{array}{l}\text { - Redes Sociales. } \\
\text { IBM. } \\
\text { - Gestión recursos. } \\
\text { Infojobs. } \\
\text { - Manantial Integra. } \\
\text { Mahou - San } \\
\text { Miguel. }\end{array}$ & $\begin{array}{l}\text { Reparalia. } \\
\text { Lilly, Ivahoe } \\
\text { Cambridge. }\end{array}$ & $\begin{array}{l}\text { - Asesoría } \\
\text { jurídica. Hogan } \\
\text { Lovells. } \\
\text { - Gestión datos. } \\
\text { Comillas } \\
\text { - Traducción. } \\
\text { Permondo } \\
\text { - Plan } \\
\text { comunicación. } \\
\text { Esade Alumni. }\end{array}$ & $\begin{array}{l}\text { - Minuto Lumiere. } \\
\text { La pierna } \\
\text { Audiovisual. } \\
\text { - Fnac: cesión } \\
\text { espacio. } \\
\text { - Difusión } \\
\text { demandas. } \\
\text { Fremap. } \\
\text { - Fnac: cesión de } \\
\text { espacios. }\end{array}$ \\
\hline
\end{tabular}

5. Los resultados del proyecto: beneficios y logros para los agentes implicados en el voluntariado corporativo con Fundación Manantial

Tras la experiencia de Fundación Manantial con diversas empresas podemos afirmar que el trabajo de voluntariado corporativo genera importantes beneficios en todos los entornos participantes (organización no lucrativa, beneficiarios de ONL, empresa, sociedad y voluntarios). Tal y como indica la Dirección General de Voluntariado y Promoción Social (Comunidad de Madrid, 2008:10) "entre las partes implicadas se producen relaciones sinérgicas e interdependientes: las comunidades más saludables y activas atraen negocios y a nuevos empleados; y además benefician a quienes viven en ellas".

En el caso que nos ocupa podemos describir algunos de los beneficios a los que nos referimos:

\section{Beneficios para la comunidad:}

- Mayor número de personas se sensibilizan con la problemática y las necesidades sociales de las personas con trastorno mental grave.

- Se facilita la difusión del trabajo que se realiza en los recursos orientados a la rehabilitación de personas con trastorno mental grave, se desmitifica al colectivo y facilita un mayor conocimiento entre la población general.

- Las empresas se convierten en nuevos agentes que potencian el cambio social, a ojos de la comunidad se convierten en más humanas, no ajenas a los problemas sociales, lo que propicia un mejor clima social y mayores oportunidades de colaboración.

Beneficios para la empresa:

- Facilita a las entidades un mayor conocimiento sobre los trastornos mentales.

- Mejora del clima laboral de la empresa y fomento del trabajo en equipo.

- Aprovechar conocimientos y vinculación de sus empleados reforzando valores internos.

- Mejora reputación institucional e imagen pública de la empresa.

Beneficios para los empleados/voluntarios:

- Se desarrolla un mayor respeto por la diversidad funcional.

- Pueden disfrutar de vivir nuevas experiencias, conocer a personas diferentes y desenvolverse en situaciones distintas, lo que produce una fuente de bienestar personal y de desafío. 
- Sentimiento de bienestar con la actividad compartida, no sólo entre los voluntarios sino en conjunto con las personas atendidas y profesionales que participan en la actividad.

\section{Beneficios para la Fundación Manantial:}

- Apoyo en costes para la puesta en marcha de actividades.

- Mayor difusión de la ONL y problemática con la que trabaja.

- Nueva fuente de voluntarios.

- La colaboración entre ONL y empresa puede facilitar a la postre otro tipo de apoyos (donaciones, subvenciones, contactos, etc.

\section{Beneficios para las personas atendidas:}

El fin último de la puesta en marcha del VC son las personas atendidas. El VC, al igual que otros tipos de voluntariado, permite crear espacios de encuentro y ocio muy similares a los que se pueden establecer entre la población general, lo que llamamos entornos normalizados, un espacio donde poder desprenderse del "rol de enfermo". Las acciones de VC permiten a las personas atendidas vivir nuevas experiencias, conocer nuevas personas y situaciones. Con ello, se posibilita que puedan experimentar sentimientos de bienestar y que deseen realizar actividades de ocio. Este deseo les ayudará a soñar y a recuperar un sentido a su vida, que tras el advenimiento de la enfermedad mental, se había perdido para muchas de las personas atendidas.

Cuando las personas con trastorno mental grave se dan cuenta de que son capaces de hacer más cosas de las que creían que podían, cosas que antes eran un límite, se sienten triunfadores y protagonistas de su propia historia.

\section{Evaluación de experiencias recientes de voluntariado corporativo en Fundación Manantial}

A partir de 2013, en el marco del Sistema de Gestión de Calidad, se ha procedimentado un sistema de evaluación de las acciones de VC que se realicen el marco de Fundación Manantial, concretado en valoraciones cuantitativas y cualitativas. En este sentido se han pasado tres tipos de cuestionarios: a los voluntarios, a los responsables de empresa y a las personas atendidas beneficiarias de la actividad.

La mejor manera de presentar el programa de voluntariado es con la voz de los propios voluntarios y quizá algunas de las expresiones que nos hicieron llegar compañeros voluntarios de Axa Todo Corazón tras una actividad compartida con Fundación Manantial:

"Hoy se me han roto todos los esquemas, tenía algunos prejuicios respecto a las enfermedades mentales y han caído tan pronto he conocido a los compañeros de la Fundación Manantial”.

"Yo creo que todas las personas buscamos lo mismo, pero no es fácil si encima del problema, les discriminamos. Los prejuicios están y gracias a este día hoy he roto uno".

"El mero hecho de ir y disfrutar una mañana trabajando juntos (...) hace que las reticencias iniciales desaparezcan sin darte cuenta”.

A modo de conclusión, el VC enriquece la vida de todas las personas y entidades que participan en él.

Tanto las personas atendidas como los voluntarios tienen ahora algo importante que contar, historias que merecen la pena ser escuchadas, saben que son capaces de hacer grandes cosas, que vale la pena implicarse en su propia vida y en su entorno. Como fundación tenemos el orgullo de poder escucharles, seguirles y apoyarles; de facilitar este escenario que promueve el cambio, que empuja a este mundo a caminar hacia la integridad. 
Actualidad económica (20II): "Voluntades que suman”, Actualidad Económica (OI-08-20I I): 69.

Cabra de Luna, M. A. (2013): Reflexiones para una estrategia que avance en la efectividad de los derechos de las personas con discapacidad $y$ sus familias (en línea). http://semanal.cermi. es/noticia/Reflexiones-estrategia-efectividadderecho-discapacidad.aspx', acceso Io de septiembre de 2013.

Carazo, J. (20I I): "Una visión global del voluntariado corporativo”, Cinco Días, Madrid (I7-IO-2OII).

Club de Excelencia en Sostenibilidad y Fundación Adecco (20IO): Estudio sobre el estado del voluntariado corporativo en España 20 Io. El valor del Voluntariado Corporativo para la empresa y sus colaboradores, Madrid (en línea). http://www.fundacionadecco.es/_data/ SalaPrensa/Estudios/pdf/ı66.pdf), acceso ro de septiembre de 2013.

Comunidad de Madrid, Consejería de Familia y Asuntos Sociales (2008): Voluntariado Apoyado por la Empresa. Guía para Entidades de Acción Social, Madrid: Comunidad de Madrid.

FEVOCAM (2OI2): Relación entre las Entidades de Acción Voluntaria (EAV) y las empresas, Madrid: FEVOCAM.

Gallardo, D. et al. (2010): “Diagnóstico del Voluntariado Corporativo en la empresa española”, Revista de Estudios Empresariales, segunda época, 2: 54-80.
García, M. T. (20I2): "El voluntariado corporativo. Un modelo de responsabilidad empresarial para el desarrollo social", CIC Cuadernos de Información y Comunicación, vol. I7: 287-302.

Gómez et al. (2012): Voluntariado Corporativo en España. Informe 20 I I (en línea). http://www. observatoriovc.org/que-hacemos/, , acceso 9 de septiembre de 2013.

Lejarazu, D. (20I2): “Empresas y ONG. Un encuentro de personas". En: FEVOCAM: Relación entre las Entidades de Acción Voluntaria (EAV) y las empresas, Madrid: FEVOCAM: 24-27.

Lemonche, P. (2OI2): “Claves para la buena relación entre las entidades de voluntariado y las empresas”. En: FEVOCAM: Relación entre las Entidades de Acción Voluntaria (EAV) y las empresas, Madrid: FEVOCAM: 28-35.

Muñoz, M. et al. (2010): "El estigma de la enfermedad mental: definición e intervención". En: Pastor, A. et al: Manual de rehabilitación del trastorno mental grave, Madrid: Síntesis: 687-7I2.

Sanz, B. et al. (2012): El voluntariado corporativo en España. Modelos y perspectivas de impacto social. ESADE. Instituto de Innovación Social (en línea). ‘http://itemsweb.esade. es/wi/research/iis/publicacions/2OI 2-O4_ VoluntariadoCorporativo.pdf, acceso 9 de septiembre de 2013.

VV. AA. (2006): Guía para promover el voluntariado desde la empresa, Barcelona: Fundación "La Caixa". 